\section{Negative nanoparticles}

\section{By C. Simone Fishburn, Senior Editor}

A simple experimental error has opened up the potential for nanoparticles to treat disease rather than be used only as payloads. By accidentally using negatively charged nanoparticles instead of neutral ones, a team from Northwestern University and The University of Sydney saw selective tagging of inflammatory monocytes for destruction. ${ }^{1}$

Their spinout, Cour Pharmaceutical Development Co. Inc., is testing the particles in myocardial infarction (MI) and plans to enter Phase I testing within a year.

Inflammatory monocytes (IMs) are a subset of immature immune cells that cause tissue damage in MI and a range of other immune-mediated diseases when they rapidly infiltrate inflamed tissue in response to an injury and differentiate into macrophages or dendrocytes.

The result is the release of proinflammatory cytokines, proteases and toxic agents such as nitric oxide.

Most attempts to dampen the inflammatory response focus on inhibiting targets such as proinflammatory cytokines. This can lead to widespread immune suppression caused by the ubiquitous expression of the cytokines. By contrast, the researchers' negative nanoparticle approach aims to neutralize IMs, which lie at the root of the damage.

\section{Accentuate the negative}

Daniel Getts, Nicholas King and Stephen Miller previously collaborated on a study of the ability of antigen-conjugated nanoparticles to induce immune tolerance. ${ }^{2,3}$ This time they teamed up to investigate how immune cells enter the inflamed brain and used nanoparticles to track the cells' movement.

Getts is CSO at Cour. King is a professor of viral immunopathology at the Sydney Medical School at The University of Sydney. Miller is a professor of microbiology-immunology and dermatology at the Northwestern University Feinberg School of Medicine.

The researchers used a mouse model of West Nile virus encephalitis to produce neuroinflammation and inadvertently gave the animals carboxylated polystyrene particles, which are negatively charged, instead of uncoated, neutral ones.

More than $60 \%$ of the infected animals survived, which was in stark contrast to the $100 \%$ lethality seen in all their previous experiments using neutral particles.

"There is no antigen, no ligand, no immune-suppressing drug. We actually found a therapeutic utility for a pure particle with no attachment," Getts told SciBX.
The negative charge was the dominant factor in protecting the animals. Positively charged particles produced some toxicity, whereas neutral particles showed no efficacy, King told SciBX.

According to Getts, the key was to fine-tune the charge on the particles to a $\zeta$-potential of about $-50 \mathrm{mV}$. $\zeta$-Potential is a measure of the surface charge used in nanoparticle characterization.

Nanoparticle-treated animals that survived showed far fewer IM-derived macrophages in the brain than animals treated with neutral particles or vehicle.

The next step was determining how the nanoparticles prevented IMs from reaching the brain. The group tracked the path of IMs in nanoparticle-treated animals and learned that a membrane-bound scavenger protein, macrophage receptor with collagenous structure (Marco; Scara2), on IMs redirects them to the spleen, where they undergo apoptosis and are eliminated.

Diversion to the spleen prevented the IMs from reaching the site of inflammation and causing damage. In splenectomized animals, the nanoparticles had no protective effect and IM numbers in the brain were comparable to
"There is no antigen, suppressing drug. We actually found a therapeutic utility for a pure particle with no attachment."

-Daniel Getts, Cour Pharmaceutical Development Co. Inc. no ligand, no immunethose in untreated mice.

Although the team is continuing to explore precisely how the nanoparticles interact with MARCO, the electrostatic interaction is most likely central to the process, Miller said. MARCO contains a positively charged collagen domain and is known to bind to polyanionic surfaces.

Getts added that Marco is the protein the team identified in mice, but there is a range of other scavenger receptors that might also be involved in the nanoparticle interaction. Thus, he said, an antibody or small molecule against MARCO probably would not be as effective as using the whole nanoparticle.

The nanoparticles improved disease outcomes in models of immunemediated conditions including MI, peritoneal inflammation, multiple sclerosis (MS), inflammatory bowel disease (IBD) and cardiac and kidney reperfusion injury.

For example, in MI the negative nanoparticles increased the heart's ejection fraction and the percentage fraction shortening compared with vehicle.

Results were published in Science Translational Medicine.

\section{Taking the IMs out of MI}

Miller said that the negative nanoparticles might offer a way to limit damage when patients with MI arrive at the hospital. "Limiting early inflammatory migration when heart cells are dying could change the course of the disease," he said.

In MI, much of the damage to myocardial tissue is caused by the initial influx of IMs to the site of infarction.

According to Matthias Nahrendorf, the use of nanoparticles to take IMs out of the picture could be a good step forward. 


\section{ANALYSIS}

"Traditionally, cardiologists thought like plumbers and would focus on the coronary artery, but we are very excited that people are finally thinking about the inflammatory response in MI," he said.

Nahrendorf is an associate

"Limiting early inflammatory migration when heart cells are dying could change the course of the disease." -Stephen Miller, Northwestern University Feinberg School of Medicine

directly.

Nahrendorf said that finding the right dose of nanoparticles might be the biggest challenge. Too many nanoparticles could overly deplete IMs and lead to immune suppression. Too few would have muted therapeutic effects. In addition, he said, the team should determine whether-and at what doses-the nanoparticles bind other phagocytic cells.

Getts, King and coauthor Rachael Terry, a postdoctoral research associate at the Feinberg School of Medicine, have filed for a patent covering the findings, and the IP is licensed to Cour.

Cour CEO John Puisis told SciBX that acute MI is the first indication the company will pursue. Cour is running IND-enabling studies in Australia and hopes to start Phase I studies within 12 months.

\section{TARGETS \& MECHANISMS}

The company also is working with groups in Nepal and other developing countries to use the technology in flavivirus infections similar to West Nile virus.

Fishburn, C.S. SciBX 7(5); doi:10.1038/scibx.2014.135

Published online Feb. 6, 2014

\section{REFERENCES}

1. Getts, D.R. et al. Sci. Transl. Med.; published online Jan. 15, 2014; doi:10.1126/scitranslmed.3007563

Contact: Nicholas J.C. King, Sydney Medical School at The University of Sydney, Sydney, New South Wales, Australia e-mail: nickk@pathology.usyd.edu.au

Contact: Stephen D. Miller Northwestern University Feinberg School of Medicine, Elmhurst, III. e-mail: s-d-miller@northwestern.edu

Contact: Daniel R. Getts, same affiliation as above e-mail: d-getts@northwestern.edu

2. Getts, D.R. et al. Nat. Biotechnol. 30, 1217-1224 (2012)

3. Lou, K.-J. SciBX 5(47); doi:10.1038/scibx.2012.1226

4. Leuschner, F. et al. Nat. Biotechnol. 29, 1005-1010 (2011)

5. Basha, G. et al. Mol. Ther. 19, 2186-2200 (2011)

6. Baas, T. SciBX 4(41); doi:10.1038/scibx.2011.1134

\section{COMPANIES AND INSTITUTIONS MENTIONED}

Cour Pharmaceutical Development Co. Inc., Elmhurst, III. Massachusetts General Hospital, Boston, Mass. Northwestern University, Evanston, III. Northwestern University Feinberg School of Medicine, Evanston, III.

Sydney Medical School at The University of Sydney, Sydney, New South Wales, Australia

The University of Sydney, Sydney, New South Wales, Australia 